\title{
Référence, document et bibliographie
}

\author{
Élisabeth Parinet \\ École nationale des Chartes (Paris)
}

Aujourd'hui, dans l'Université française, on ne conçoit pas une thèse en lettres ou en sciences humaines sans une volumineuse bibliographie, qui pourra éventuellement faire l'objet de remarques, voire de questions au moment de la soutenance. Quel rôle assigne-t-on à cet exercice obligé qui n'a jamais fait l'objet d'une définition officielle ? La bibliographie d'une thèse est-elle la liste des ouvrages que l'auteur a lus ? Dans ce cas, la masse des dépouillements agit-elle comme la garantie du travail accompli, du temps passé, et la sélection opérée par son auteur révèle-t-elle des choix intellectuels dont le jury pourra discuter ? Si la bibliographie s'adresse au public plus large des lecteurs et des chercheurs intéressés par le sujet, quel est son 
but ? Suggérer des lectures ? Préciser des références d'ouvrages cités en note? Le flou entourant la pratique actuelle des bibliographies de thèse est-il lié à l'accroissement rapide de la masse documentaire qui rend l'exhaustivité impossible? Comment cet exercice obligé s'est-il imposé et quel but se fixaitil à l'origine?

\section{Une pratique balbutiante}

Les thèses ne représentent, au XIX ${ }^{\text {e }}$ siècle, qu'une très faible part des travaux historiques et littéraires. S'en distinguent-elles par une exigence particulière concernant la bibliographie ? Il ne le semble pas.

Pour ce qui est des travaux historiques qui ne sont pas universitaires, on constate que les auteurs du XIXe siècle font une place très faible à la bibliographie. Pas plus Michelet qu'Augustin Thierry ou Guizot ne donnent en annexe une bibliographie des ouvrages qu'ils ont pu consulter. Non qu'ils prétendent n'avoir eu aucun prédécesseur. Michelet, dès la première page de son Histoire de France, avoue : "Mon livre (la critique la plus sévère en conviendra) est sorti tout entier des sources originales. Cependant, je dois beaucoup à quelques-uns de nos contemporains ». Avec les quatre historiens qu'il cite (Sismondi, Amédée et Augustin Thierry, et surtout Guizot), il reconnaît qu'il s'agit de «parenté intellectuelle», de «communion de pensée». Ceux envers qui il a une dette documentaire sont évoqués immédiatement, mais en note :

Je reconnaîtrai en temps et lieu mes obligations envers ceux qui ont traité avec un mérite supérieur, quelque partie de notre histoire politique ou littéraire. Je dois nommer, entre autres, les savants continuateurs des Bénédictins, et mes collègues de la 
Société des antiquaires de Normandie. J'aurai aussi occasion de dire tout ce que je dois à plusieurs savants étrangers, J. Grimm, Gans, etc. (p. V-VI).

Cette « occasion » lui sera donnée par les notes infrapaginales. Des trois catégories de notes dont Michelet émaille son texte (références des citations et des sources, commentaires marginaux, renvois à d'autres ouvrages), c'est la dernière, celle qui nourrirait aujourd'hui une bibliographie, qui est la moins représentée. Encore l'historien se réfère-t-il souvent au même ouvrage : c'est le cas de l'Histoire des Gaulois d'Amédée Thierry, à laquelle Michelet renvoie dès la page 7 du tome 1 et qui sera encore plusieurs fois citée dans ce volume. En tout, dans ce volume consacré aux Gaulois, Michelet renverra à une dizaine d'ouvrages historiques récents.

Michelet avait introduit, d'entrée de jeu, une hiérarchie entre ses «dettes» envers les historiens qui l'ont précédé, privilégiant ceux avec qui il se sent des affinités intellectuelles. Il s'agit moins, pour lui, de se réclamer d'une école historique encore que les contemporains ne s'y trompent pas - que de faire connaître l'homme qui entreprend cette nouvelle histoire de France et sa démarche intellectuelle. Enfin, on notera que, très normalement, après avoir affirmé l'importance des «sources originales» dans son discours historique, Michelet rend hommage aux travaux des érudits qui, au sein des sociétés savantes locales ou dans le cadre des vastes entreprises nationales auxquelles Guizot a fourni son appui, produisent un énorme travail d'édition de sources. Sur ces deux points, nous reviendrons.

Tous les historiens du XIX ${ }^{\text {e }}$ siècle ne disent pas aussi explicitement leur reconnaissance à certains de leurs 
prédécesseurs, mais l'exemple de Michelet est suivi par beaucoup de ses confrères. Ainsi, Adolphe Chéruel ouvre son Histoire de France pendant la minorité de Louis XIV en reconnaissant: "Les événements que retrace cet ouvrage ont été l'objet de travaux considérables en France et à l'étranger. Pour ne parler que des plus récents... »Et il cite trois ouvrages français «dont le mérite a été apprécié par les juges les plus compétents » et deux ouvrages allemands qui ont traité du sujet «avec beaucoup de sagacité et d'érudition» (vol. 1, p. ii). Et le sujet est clos.

Cette place très mineure accordée à la bibliographie du sujet est-elle liée au souci de ne pas alourdir un ouvrage destiné à un public qui n'est pas spécialiste? Quand il s'adresse à ses pairs, l'historien du XIXe siècle est-il plus explicite sur ses modèles et ses lectures ? Qu'en est-il, par exemple, des thèses soutenues à l'université de Paris ? La thèse est au XIX'e siècle et au $\mathrm{XX}^{\text {e }}$ siècle le passage obligé pour accéder à un poste de professeur dans l'Université. Si la création de la thèse de lettres date des années 1806-1808, elle ne présente pas d'intérêt pour notre sujet jusqu'aux années 1850 ; elle est jusque-là un exercice purement formel, rhétorique, avant tout oral, puisque, jusqu'en 1840, le candidat n'imprime que le programme de sa soutenance qui est une sorte d'événement universitaire et mondain. « Le doctorat même ne suppose aucune connaissance acquise en dehors du lycée [...]. Entre le mérite d'un bachelier ès lettres et celui d'un docteur ès lettres, il n'y a qu'un degré de virtuosité de plus », juge Alphonse Aulard, qui soutint la sienne bien plus tard (cité dans Jolly, 1993, p. 333-334)... En 1840, la réforme de la thèse oblige le candidat à imprimer le texte-même de sa thèse. Toutefois, ces textes dépassent rarement une cinquantaine de pages et ne s'écartent guère du texte prononcé. 
Puis elles s'étoffent lentement. Entre 1850 et 1870, elles comptent entre 100 et 200 pages format in- $8^{\circ}$, ce qui implique une évolution de fond : ce n'est plus un exercice de rhétorique, mais une véritable étude historique ou littéraire. C'est à partir de ce moment que les thèses nous intéressent.

Deux constations s'imposent dès l'abord. Aucune n'offre de bibliographie, au sens d'une liste d'ouvrages présentée en annexe. Toutes, ou presque, usent de la note infrapaginale, mais avec modération. Au-delà de ces caractéristiques communes, il faut distinguer entre sujets littéraires et sujets historiques. Les littéraires n'usent de la note de bas de page que pour donner la référence des citations faites dans le corps du texte. C'est le cas, par exemple, de J. A. Lisle qui, le 2 novembre 1852, reçoit du Recteur le permis d'imprimer sa thèse de 126 pages, intitulée Essai sur les théories dramatiques de Corneille d'après ses discours. La thèse de Taine, présentée en avril 1853, Essai sur les Fables de La Fontaine, ne comporte même aucune note au long de ses 200 pages ; les références des citations sont intégrées au texte-même. Ni l'un ni l'autre n'évoquent, au cours de son analyse, la lecture d'ouvrages d'autres critiques.

Les historiens ont déjà une pratique un peu différente, bien que les usages soient loin d'être codifiés. Certains, tels Fustel de Coulanges, se contentent de donner en notes les références des textes cités. À l'inverse, la thèse de Victor Duruy, État du monde romain vers le temps de la fondation de l'Empire, soutenue en 1853, comporte de nombreuses notes de bas de page aux usages variés. Certaines contiennent, comme de juste, les références des textes cités; d'autres apportent des informations complémentaires comme une brève chronologie de la conquête de l'Espagne par les Romains ; d'autres encore 
sont un lieu d'échanges, commentaire adressé au lecteur ou présentation des opinions d'autres historiens. On prendra en exemple la note 1 de la page 10: "Il est vrai que, suivant Masdeu, Historia critica de Espana, les Trudetans comptaient leurs années en saisons, ce qui ramèneraient ces 6000 ans à une antiquité moins problématique, environ 1500 ans avant Jésus-Christ. Cf. Rossew Saint-Hilaire, Hist. d'Esp., tome 1 ». Surtout, il est à souligner que la thèse de Duruy est complétée d'un «Appendice» ainsi justifié : «J'ai indiqué dans le courant de ce travail beaucoup d'ouvrages modernes que j'ai consultés ; je dois ici une mention particulière à quelques-uns ». Suivent deux pages d'une bibliographie commentée, riche de 20 titres :

Les travaux de M. Letronne sur l'Égypte, de M. Laboulaye, [...], les Germains de M. Ozanam, [...] l'Histoire d'Espagne de M. Rosseuw Saint-Hilaire, le livre qui a versé tant de faits et d'idées dans notre littérature historique [...], celui de M. Wallon sur l'Esclavage qui est d'une érudition si française, enfin la riche masse des Mémoires de l'Académie des Inscriptions m'ont fourni de précieux renseignements (p. 262).

Entre ces deux exemples extrêmes que représentent Fustel de Coulanges et Victor Duruy, le plus grand nombre se contentent de dire ce qu'ils doivent à quelques ouvrages. Dès les premières lignes de son introduction, Hippolyte Dansin avoue: «Les institutions et les réformes de l'ancienne monarchie ont été étudiées depuis quelques années avec un soin particulier» (p. 208). Et de citer, en note, quatre ouvrages. Il rendra hommage, un peu plus loin à l'un de ses confrères : "Nous n'entrons dans aucun détail sur Jacques Cœur pour ne pas répéter des détails qui ne seraient plus neufs depuis le savant travail de M. Pierre Clément sur ce financier... » (p. 79). Cette façon discrète de glisser quelques références bibliographiques est fréquente. Ainsi, avant de commencer sa biographie de 
Sadolet, A. Joly écrit: "C'est aussi que des hommes d'opinions les plus diverses, philosophes ou catholiques ardents, ont tour à tour glorifié le nom de Sadolet» (p. 222). Ce qui est une façon d'annoncer la liste de sources imprimées qui figure en note.

Enfin, à travers ces quelques citations, on voit qu'aucune règle ne préside non plus à la présentation des références bibliographiques : si le nom de l'auteur et le titre sont le plus souvent présents, l'année de publication de l'ouvrage est rarement indiquée ; le tome l'est parfois, exceptionnellement la page. Il est évident qu'aucune règle implicite ou explicite ne régit la présentation ni même l'existence de la bibliographie dans la thèse universitaire.

Jusqu'aux années 1870, les travaux universitaires ne se distinguent donc pas, sur le sujet de la bibliographie, des autres travaux historiques. Les auteurs sont libres de rendre hommage comme ils le veulent à leurs prédécesseurs. Le plus souvent, quelques lignes dans l'introduction et quelques notes infrapaginales suffisent à distinguer les ouvrages les plus intéressants. L'auteur gratifie généralement l'historien cité d'une appréciation élogieuse. "Voyez le bel article inséré par M. Ampère dans la Revue des Deux-mondes, $1^{\mathrm{er}}$ août 1833 ", conseille Michelet à qui s'intéresse à la légende de Siegfried. Il est extrêmement rare que l'ouvrage cité fasse l'objet d'une critique. «M. Saint-René Taillandier a tiré une fort belle biographie [de Maurice de Saxe], savante, curieuse, intéressante (Revue des Deux Mondes, 1864). Seulement, il me semble un peu trop favorable à ce héros de second ordre que la fortune a tant favorisé, exagéré, surfait » (p. 87). Encore Michelet adresse-t-il la critique moins au biographe qu'à son héros. 


\section{Vers la normalisation de la bibliographie (1880-1914)}

Bien que les thèses soient devenues progressivement beaucoup plus volumineuses ${ }^{1}$ (le décret du 28 juillet 1903, qui définit le contenu scientifique de la thèse, met d'ailleurs en garde contre une inflation du texte), la présence d'une bibliographie n'y a toujours rien d'obligatoire et sa présentation peut être assez désinvolte.

Dans l'introduction de sa thèse publiée en 1882, Le régime féodal en Bourgogne jusqu'en 1360, Charles Seignobos cite longuement ses sources imprimées et ajoute une rubrique «Travaux modernes» ainsi introduite : «On a consulté aussi plusieurs recueils, histoires locales et monographies ». Suivent quelques ouvrages désignés par le nom seul de l'auteur et le titre, sans autre indication. En 1903, Albert Mathiez, lui, met en tête de sa volumineuse thèse consacrée à la théophilanthropie pendant la Révolution un chapitre intitulé "Sources imprimées » dans lequel il décrit les différentes catégories de sources (pamphlets, journaux, etc.) qu'il a consultées et termine ainsi : "Dans ces derniers temps, enfin, des érudits provinciaux ont commencé à écrire quelques monographies sur l'histoire des cultes révolutionnaires dans leur département» (p. 722). On les retrouvera dans les notes au fil du texte.

À côté de ces historiens que l'on attendait plus diserts sur leurs lectures préparatoires, on rencontre des auteurs plus explicites. La thèse de Romain Rolland, soutenue en 1895, se clôt sur une bibliographie de plus de 120 références mêlant, sur

1 Albertini (1992, p. 110) estime leur volume à 500 pages en moyenne autour de 1900. 
dix pages, sources imprimées, ouvrages critiques récents et ouvrages généraux (dictionnaire, histoire de la musique, etc.).

Si une grande liberté est donc laissée aux auteurs de thèses, on constate néanmoins que les indications bibliographiques données en annexe sont de plus en plus fréquentes. Grâce au recensement des thèses de Mourier (1880), puis de Maire (1903), il est possible de repérer la présence éventuelle de ces annexes. À partir de 1867, elles accompagnent quelques thèses, sous des noms divers : «notice bibliographique», "liste des ouvrages à consulter», "note bibliographique», «index bibliographique», etc. Avant 1885, on n'en compte toutefois qu'une dizaine parmi les thèses soutenues à Paris. Puis, elles prennent le simple titre de «bibliographie » et se font plus nombreuses, placées en tête ou, après 1895 , à la fin des thèses.

Les littéraires sont les moins nombreux à se plier à l'exercice. Ni Jules Lemaître (La comédie après Molière, 1883), ni Gustave Lanson (Nivelle de La Chaussée, 1887), ni Eugène Lintilhac (Beaumarchais, 1888) ne font place à une bibliographie. Seul Gustave Larroumet en donne une, en 1882, dans sa thèse sur Marivaux.

\section{Bibliographie et historiographie}

Les historiens de la Monarchie de juillet renouvellent le discours historique grâce à l'exploitation de sources pour beaucoup inédites. Ils se doivent de citer ces sources, en utilisant pour cela la note de bas de page, et se trouvent redevables tant aux grandes entreprises nationales d'édition de sources qu'au travail plus modeste des érudits locaux. Les 
grands chantiers ouverts au XIX siècle imposent progressivement l'idée d'un travail collectif de la communauté historienne, même si chaque historien se considère seul responsable de son discours historique. Les historiens sont conscients de l'accumulation nécessaire des connaissances. L'apparition de références bibliographiques traduit cette évolution. L'exemple de Michelet montre que le processus est enclenché, mais en même temps, il reste timide : l'hommage est rendu de façon globale, car l'auteur assume la responsabilité de l'ensemble de son travail (il faut dire que beaucoup ont, non sans raison, le sentiment d'être pionniers dans leur domaine). Il est maître de ses choix documentaires et s'arroge le droit de distribuer les louanges à ceux de ses confrères dont il a particulièrement estimé le travail. On est donc très loin d'une bibliographie exhaustive.

En revanche, le critique littéraire se perçoit comme seul face au texte ou à la pensée de l'auteur étudié. Malgré les différences de méthode et l'influence sur les littéraires du discours positiviste, chaque étude reste la rencontre d'un critique avec un écrivain et une œuvre. Il est logique, dans ces conditions, que les littéraires s'exemptent de bibliographies. Il faut attendre Gustave Lanson pour voir affirmer de manière forte la valeur de l'objectivité en matière d'étude littéraire. « Notre idéal est d'arriver à construire le Bossuet ou le Voltaire que ni le catholique ni l'anticlérical ne pourront nier, de leur en fournir les figures qu'ils reconnaîtront pour vraies et qu'ils décoreront ensuite comme ils voudront de qualificatifs sentimentaux » (p. 178). Cet idéal d'objectivité, les historiens se le sont déjà fixé depuis plusieurs années et les littéraires ne pouvaient rester à l'écart du mouvement. Il a pour conséquence de rendre nécessaire la confrontation des points de vue et, 
donc, la reconnaissance du rôle joué dans l'élaboration de l'analyse par les réflexions des prédécesseurs.

Un autre facteur décisif est l'orientation vers une histoire littéraire qui rend l'alignement des méthodes, des objectifs et des moyens sur ceux des historiens plus nécessaire. Si l'on ajoute à cela le désir de Lanson d'étendre aux textes modernes les méthodes philologiques appliquées aux textes antiques et, grâce à Gaston Paris, aux textes médiévaux, pour développer les éditions de textes et les recherches de sources inédites, on comprend pourquoi les bibliographies vont devenir habituelles dans les travaux universitaires littéraires et prendre une ampleur nouvelle.

Ce développement des bibliographies dans les travaux scientifiques n'est évidemment pas sans rapport avec le travail bibliographique qui est fait dans les revues. Plus étroitement spécialisée que la Revue critique d'histoire et de littérature de Paul Meyer, qui accumulait déjà les comptes rendus depuis 1866, dès son premier numéro, paru en 1894, la Revue littéraire de la France fait une large place à la bibliographie : comptes rendus de publications, dépouillement de périodiques littéraires, liste des livres nouveaux qui n'ont pas fait l'objet d'un compte rendu, etc. À défaut d'avoir l'organisation qu'on lui connaît aujourd'hui, la bibliographie de la $R H L F$ en a déjà le contenu. Ce développement d'un outil de travail bibliographique est significatif d'un changement dans les méthodes de travail. Néanmoins, les littéraires sont en retard sur les historiens qui, avant 1914, auront déjà publié plusieurs bibliographies de l'histoire de France (Bibliographie de l'histoire de France de Gabriel Monod, Bibliographie des travaux publiés de 1866 à 1897 sur l'histoire de la France depuis 1789 de Pierre 
Caron, Répertoire méthodique de l'histoire moderne et contemporaine de la France de Caron et Brière, etc.), alors que le Manuel bibliographique de Lanson ne paraîtra qu'en 1921.

\section{Les « damnés de la thèse » et la bibliographie}

La bibliographie des thèses d'État va devenir un monstre dans les années 1950. Il faut dire que la thèse elle-même est devenue, elle aussi, une entreprise gigantesque, mobilisant le chercheur pendant dix ans au moins de sa carrière. Devant l'afflux des candidats, l'Université française a trouvé ce moyen de sélectionner sinon les meilleurs, du moins les plus résistants de ses chercheurs. Les thèses en sciences humaines se prêtent particulièrement à cette dérive, si l'on en croit Emmanuel Le Roy Ladurie à qui nous laisserons la responsabilité de cette explication: "Comme il est impossible dans les sciences humaines de définir des critères de qualité admis par tous, on a valorisé, pour bien des raisons, la quantité » (p. 540).

Pour ce monument qui ouvre les portes de l'Université, le candidat se doit de ne rien négliger et la bibliographie peut constituer un volume entier de la thèse. Ainsi, les thèses de futurs professeurs de la Sorbonne, comme Marie-Jeanne Durry ou Raymond Picard, se terminent pas un volume entier de bibliographie. La thèse de Raymond Picard, consacrée à la carrière de Racine, offre un inventaire des sources si important qu'il donnera lieu à une publication autonome aux éditions des Belles Lettres quand l'auteur publiera le texte de sa thèse dans la Bibliothèque des idées chez Gallimard, en 1956. Quant aux ouvrages consacrés à Racine, Picard renonce à en donner une bibliographie exhaustive dans ce volume de près de 700 pages : 
pour les publications anciennes, il renvoie à des bibliographies littéraires déjà publiées (Lanson, Höhlfeld, Williams); pour celles de la période 1940-1955, le lecteur les trouvera rassemblées dans un "état présent des études raciniennes » qu'il a publié dans L'Information littéraire et le volume publié chez Gallimard ne retiendra "que » les ouvrages cités dans le texte, soit tout de même, 200 références environ. La thèse de Raymond Picard n'est qu'un exemple parmi d'autres. Vingt ans plus tard, quand il publie sa thèse consacrée à l'éloquence à l'âge classique, Marc Fumaroli l'assortit d'une bibliographie de 1722 références...

La soutenance solennelle, qui dure plusieurs heures, permet un échenillage méticuleux du texte de la thèse, $\mathrm{y}$ compris de sa bibliographie. C'est dans cette pratique que l'auteur de Montaillou voit la cause de cette inflation bibliographique : «L'auteur de la thèse pour désamorcer toute critique possible en cours de soutenance sera donc incité à farcir son exemplaire définitif d'un nombre encore plus grand de notes infra-paginales, de références bibliographiques ou érudites, dont l'utilité n'est pas toujours évidente et qui parfois confinent, hélas!, à la cuistrerie » (Le Roy Ladurie, 1973/1978, vol. 2, p. 541).

L'explication est un peu courte, même si elle contient une part de vérité. L'exemple de la thèse de Raymond Picard montre bien que plusieurs phénomènes se conjuguent pour aboutir à cette croissance exponentielle des bibliographies: l'exigence lansonienne d'une recherche de sources toujours plus nombreuses; l'augmentation du nombre des publications savantes et la multiplication des bibliographies spécialisées qui les enregistrent; l'extension des études littéraires en direction 
d'une histoire de la société, d'une histoire des idées, d'une histoire culturelle (selon les époques) qui entraîne son lot de lectures supplémentaires. Ainsi, dans la sélection qu'il opère pour la publication de sa thèse chez Gallimard, Raymond Picard, tout en se réclamant de Sainte-Beuve, Jules Lemaître, Daniel Mornet, Gustave Larroumet, etc. retient aussi François Simiand pour son étude sur le mouvement des prix des denrées.

Encore Raymond Picard est-il visé par Roland Barthes dans la polémique qui se développe en 1960 dans la revue Annales. Il est de ces historiens de la littérature à qui Barthes reproche de n'avoir pas choisi clairement d'être historien, d'avoir négligé une approche sociologique, psychanalytique, psychologique, et d'avoir méconnu les travaux de Lucien Goldmann, Georges Poulet, Jean Starobinski, Charles Mauron, etc. Barthes, lui-même, n'assortit ses textes sur Racine que de très brèves indications bibliographiques, qui sont des moyens de se réclamer de "parentés intellectuelles», pour reprendre Michelet.

Barthes ne fait que suivre un mouvement déjà observable chez d'autres chercheurs avant lui. Tandis que la masse des écrits à citer s'accroît démesurément, on voit certains historiens opérer un discret repli.

Lucien Febvre en est un exemple, dans son étude sur la religion de Rabelais. Sa bibliographie, dont Henri Berr, dans la préface, dit qu'elle est « un modèle - pour la sélection et le classement», commence par un avertissement: «Les indications bibliographiques qui vont suivre ne constituent pas la bibliographie exhaustive d'un sujet difficile à cerner. Nous n'avons retenu pour les citer que les livres dont nous nous sommes effectivement servi [...]» (p. 504). Encore faut-il 
s'entendre sur ce qui a «servi». Une large partie de la bibliographie établie par Febvre sera, en effet, consacrée à "l'époque de Rabelais », à l'histoire des idées, aux savants, à la vie intellectuelle, et fera place à Bloch, Huizinga, Gilson, LévyBruhl, etc. Certains de leurs écrits n'ont pas un rapport direct avec Rabelais, mais sont des outils de la réflexion: L'âme primitive, La mentalité primitive, Le surnaturel chez les primitifs de Lévy-Bruhl, par exemple. Fernand Braudel a plus de raisons encore de reculer devant une bibliographie exhaustive de son sujet, mais il justifie ainsi son choix : "Les sources du présent livre sont avant tout manuscrites. L'abondante littérature du sujet n'a été utilisée qu'après enquête dans les autres, pour les compléments, la coordination des résultats, les indispensables contrôles » (p. 523).

Ce n'est pas un hasard si les historiens de l'école des Annales, puis ceux de la Nouvelle Histoire comme Le Roy Ladurie marquent plus que d'autres leur lassitude devant l'exercice de construction d'une bibliographie exhaustive. Outre que l'exhaustivité devient irréalisable, il est important pour eux de faire ressortir par la bibliographie leur rupture avec des méthodes, des écoles de pensée, et d'affirmer leur connivence avec d'autres chercheurs de la même mouvance intellectuelle.

Les jugements sans indulgence portés par Le Roy Ladurie sur la thèse et sa bibliographie ont une origine double. D'une part, ils font partie d'une remise en cause globale de la pratique de la thèse d'État qui paraît dans le journal Le Monde, en 1968, dans le grand bouleversement de l'après-mai que traverse l'Université. Sous le titre humoristique «Apologie pour les damnés de la thèse », Le Roy Ladurie s'interroge sur l'utilité de la thèse, qu'il accuse de stériliser la recherche en privilégiant un 
travail de longue haleine épuisant au détriment de travaux plus courts, plus nombreux et plus incisifs qui stimuleraient davantage la réflexion de la communauté des chercheurs. La critique fera long feu puisqu'il faudra attendre 1984 pour voir naître le nouveau doctorat. Entre temps, tout en réaffirmant la prééminence du doctorat d'État, les textes inviteront les conseils d'université à "fixer une limite maximum à la dimension de la thèse », supprimeront la thèse complémentaire et admettront la thèse soutenue sur un ensemble de travaux. Pour ce qui est de la bibliographie de thèse, le texte de Le Roy Ladurie suggère son inutilité, l'épreuve de la soutenance passée, et le gâchis du temps utilisé à la bâtir. D'autre part, l'auteur se rattache à ce courant d'historiens qui veulent affirmer leur appartenance à une famille de pensée. Non que Le Roy Ladurie ait tout à fait renoncé à faire suivre sa thèse d'une bibliographie classique. La version de ses Paysans $d u$ Languedoc, publiée par Mouton, compte 84 pages de bibliographie, soit près de 3000 titres ! Elle est classée par ordre alphabétique strict de noms d'auteurs et on y trouve des instruments de travail classiques comme le Dictionnaire des postes ou le Dictionnaire d'Expilly. On ne s'étonnera pas non plus d'y trouver de nombreux travaux de Pierre Goubert ou de Roland Mousnier. En revanche, il n'allait pas de soi qu'y figurent Freud (pour quatre titres), Soboul (Les sans-culottes parisiens de l'an II), Michel Foucault, Claude Lévi-Strauss (Tristes tropiques, Anthropologie structurale), Bourdieu (appelé Claude par erreur) pour Sociologie de l'Algérie... La bibliographie se fait «militante » : l'historien affiche la famille de pensée à laquelle il se rattache. Dans le cas de Le Roy Ladurie, dont l'introduction se clôt avec la revendication d'une "histoire totale», elle accompagne logiquement le sentiment de rupture. Quand il 
n'est plus dépendant des règles implicites de la thèse, Le Roy Ladurie compose une bibliographie resserrée pour Montaillou, village occitan, qui fait l'économie des ouvrages historiques classiques pour ne plus citer, dans l'espace qui est alloué à la bibliographie dans un volume de la Bibliothèque des histoires, que des historiens de la Nouvelle histoire et, encore, Georges Balandier...

Le retour à une bibliographie de thèse qui serait un manifeste intellectuel fait long feu. La pratique actuelle, aussi bien chez les historiens que chez les littéraires, est toujours celle d'une bibliographie volumineuse, sélective par force et prudemment consensuelle, dans la majorité des cas. Peut-il en être autrement quand les autres publications, en lettres ou sciences humaines, offrent, elles aussi, des bibliographies dont le volume est proportionnel à l'ambition érudite de l'ouvrage ? Toutefois, l'évolution des instruments de travail mis à la disposition des chercheurs et, en particulier, la multiplication des catalogues numérisés, des bibliographies et des revues électroniques rendent plus aisé le repérage de la documentation. La fonction de signalement d'ouvrages utiles au sujet que remplissait la bibliographie de thèse devrait perdre de son utilité. Lui resterait comme rôle de marquer les étapes du cheminement intellectuel qui sous-tend la thèse. Une sorte de retour aux sources? 


\section{Bibliographie}

AlBertini, Pierre. 1992, L'école en France, Paris, Hachette.

AULARD, Alphonse. 1911, Napoléon Ier et le monopole universitaire. Origines et fondements de l'Université impériale, Paris.

BARTHES, Roland. 1960, " Histoire ou littérature », Annales, n 3 ; repris dans Sur Racine, Paris, Seuil, coll. « Pierres vives », 1963.

BRAUDEL, Fernand. 1966, La Méditerranée et le monde méditerranéen au temps de Philipe II, Paris, Armand Colin.

CHÉRuEL, Adolphe. 1879, Histoire de France pendant la minorité de Louis XIV, Paris, Hachette.

Coulanges, Fustel de. 1858, Polybe ou la Grèce conquise par les Romains, Amiens, imp. de T. Jeunet.

DANSIN, Hippolyte. 1856, Étude sur le gouvernement de Charles VII, Strasbourg, imp. de G. Silbermann.

DURUY, Victor. 1853, État du monde romain vers le temps de la fondation de l'Empire, Paris, Hachette.

FEBVRE, Lucien. 1942, Le problème de l'incroyance au XVIe siècle. La religion de Rabelais, Paris, Albin Michel.

FumARoLI, Marc. 1980, L'âge de l'éloquence: rhétorique et res literaria, de la Renaissance au seuil de l'époque classique, Paris, Champion.

Joly, A. 1856, Étude sur Sadolet, Caen, typ. de A. Hardel.

Jolly, Claude. 1993, "La thèse de lettres au XIXe et au XXe siècles : les principaux textes législatifs et réglementaires", dans Éléments pour une histoire de la thèse, Paris, Klincksieck. 
LE RoY LADURIE, Emmanuel. 1966, Les Paysans du Languedoc, Paris/La Haye, Mouton.

—. 1968, "Apologie pour les damnés de la thèse », Le Monde, 19 septembre ; repris dans Le territoire de l'historien.

—. 1973 et 1978, Le territoire de l'historien, Paris, Gallimard, 2 vol.

-. 1978, Montaillou, village occitan de 1294 à 1324, Paris, Gallimard.

MAIRE, Albert. 1903, Répertoire alphabétique des thèses de doctorat ès lettres des universités françaises, 1810-1900, avec table chronologique par université et table détaillée des matières, Paris, A. Picard et fils.

MATHIEZ, Albert. 1903, La Théophilanthropie et le culte décadaire (1796-1801). Essai sur l'histoire religieuse de la Révolution, Paris, Alcan.

MichELET, Jules. 1833, Histoire de France, Paris, Hachette.

Mourier, Louis Athénaïs et Deltour, Félix. 1880, Notice sur le doctorat ès lettres, suivie du catalogue et de l'analyse des thèses latines et françaises admises par les facultés des lettres depuis 1810, Paris, Delalain.

PICARD, Raymond. 1956, Corpus Racinianum. Recueil-inventaire des textes et documents du XVIIe siècle concernant Jean Racine, Paris, Éditions des Belles Lettres.

RoLLAND, Romain. 1895, Les origines du théâtre lyrique moderne. Histoire de l'opéra en Europe avant Lulli et Scarlatti, Thorin.

ThIERry, Amédée. Histoire des Gaulois, dans Jules Michelet, Paris, Hachette. 


\title{
Résumé
}

Aujourd'hui, dans l'Université française, une thèse en lettres ou en sciences humaines est nécessairement accompagnée d'une volumineuse bibliographie, qui pourra éventuellement faire l'objet de remarques, voire de questions au moment de la soutenance. Quel rôle assigne-t-on à cet exercice obligé qui n'a jamais fait l'objet d'une définition officielle? La bibliographie d'une thèse est-elle la liste des ouvrages que l'auteur a lus ? Si la bibliographie s'adresse au public plus large des lecteurs et des chercheurs intéressés par le sujet, son but est-il de suggérer des lectures? Préciser des références d'ouvrages cités en note? Comment cet exercice obligé s'est-il imposé et quel but se fixaitil à l'origine?

\begin{abstract}
Presently, in a French University, a PhD thesis in Humanities must provide, as an annex, an extensive bibliography. This annex may be subjected to examination, revisions, and criticisms and may, per se, receive the attention of the committee during the defence. But what is the function of this mandatory exercise? Is it simply a list of the sources cited, consulted, and read by the author of the thesis? Or is it a compendium of suggested readings for anyone interested in the topic, as the author is addressing a larger audience than the committee including researchers and readers? Or, furthermore, is it the development of footnote references? The paper follows the history of this mandatory practice, at its very origins.
\end{abstract}

